\title{
Double papilla of Vater: a rare anatomic variant
}

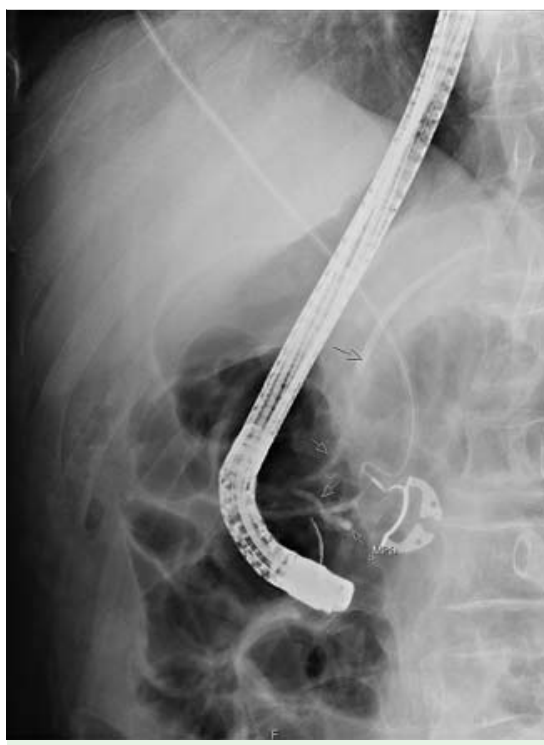

Fig. 1 Pancreatography following contrast injection into the lower papilla at the beginning of the endoscopic retrograde cholangiopancreatography (ERCP) procedure showing contrast within the main pancreatic duct (MPD).

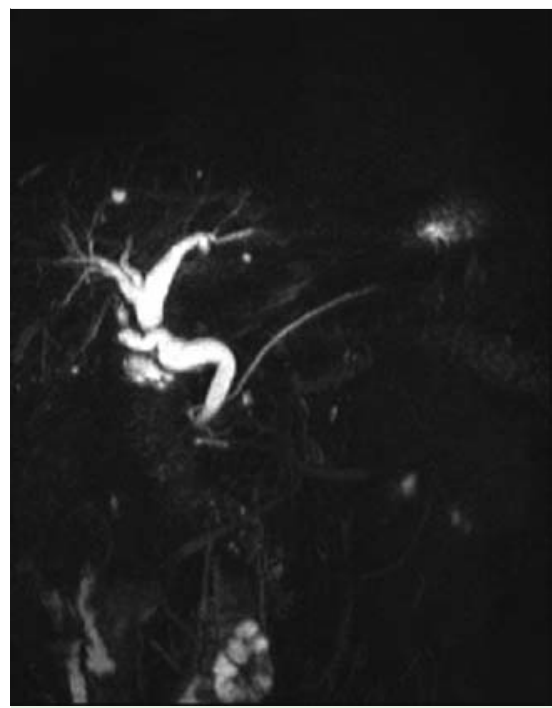

Fig. 2 Magnetic resonance cholangiopancreatography (MRCP) showing the two separate ducts.

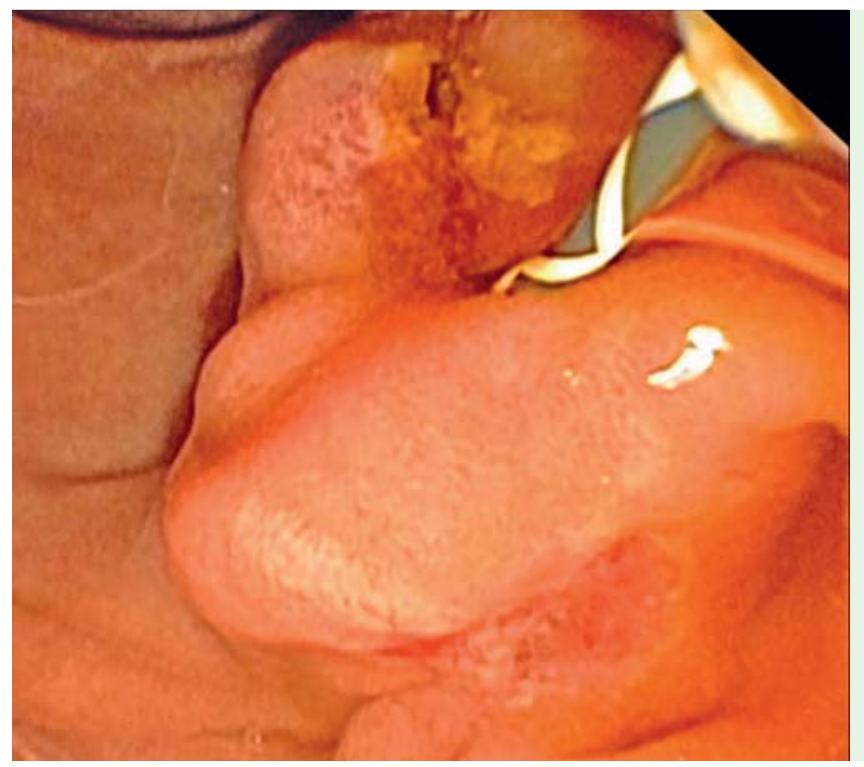

Fig. 3 Endoscopic view showing the double papilla after cannulation and sphincterotomy of the upper orifice (the common bile duct [CBD]).

A 68-year-old man who had undergone a cholecystectomy in the 1980 s was admitted acutely with right upper quadrant pain. He was treated conservatively and an ultrasound scan showed mild common bile duct (CBD) dilatation and one stone. A magnetic resonance cholangiopancreatography (MRCP) scan was subsequently performed, which visualized a 9-mm stone in the CBD. After discussion with the patient, it was decided to perform an elective endoscopic retrograde cholangiopancreatography (ERCP).

During the ERCP, the papilla was identified in the usual position, this being almost $1 \mathrm{~cm}$ below what was thought initially to be a hypertrophic minor papilla. Cannulation proceeded very easily by means of a sphincterotome over a 0.025-inch guidewire and contrast injection was performed. Surprisingly the pancreatic duct was visible during fluoroscopy, which was repeated three times ( $\nabla$ Fig. 1 ). We decided to review the MRCP images together with the radiologist. Thorough examination of the images showed an unexpected finding: the pancreatic duct had a totally separate orifice below the CBD orifice via what could indeed represent a double papilla (๑ Fig. 2).
We changed the position of the scope and the sphincterotome and cannulated the upper orifice, which was previously considered to be a large minor papilla ( $\bullet$ Fig.3). Contrast injection revealed the mildly dilated CBD and the stone ( $\bullet$ Fig.4). A sphincterotomy was performed and was followed by balloon stone extraction. Immediate bleeding was treated by means of a fully covered metal stent (WallFlex; Boston Scientific, Natick, Massachusetts, USA).

The patient did well after ERCP with only mild elevation of his amylase level and was discharged home the following day in good general condition. He underwent a further ERCP 6 weeks later for stent extirpation and has done well, with no problems, since the procedure ( $\bullet$ Video 1$)$. In conclusion, this rare anatomic variant led in this patient to undesirable pancreatic duct cannulation, which carries a well-known risk for post-ERCP pancreatitis (PEP). We believe awareness of this condition could help endoscopists to avoid PEP in their patients.

\section{Endoscopy_UCTN_Code_CCL_1AZ_2AK}

Competing interests: None 

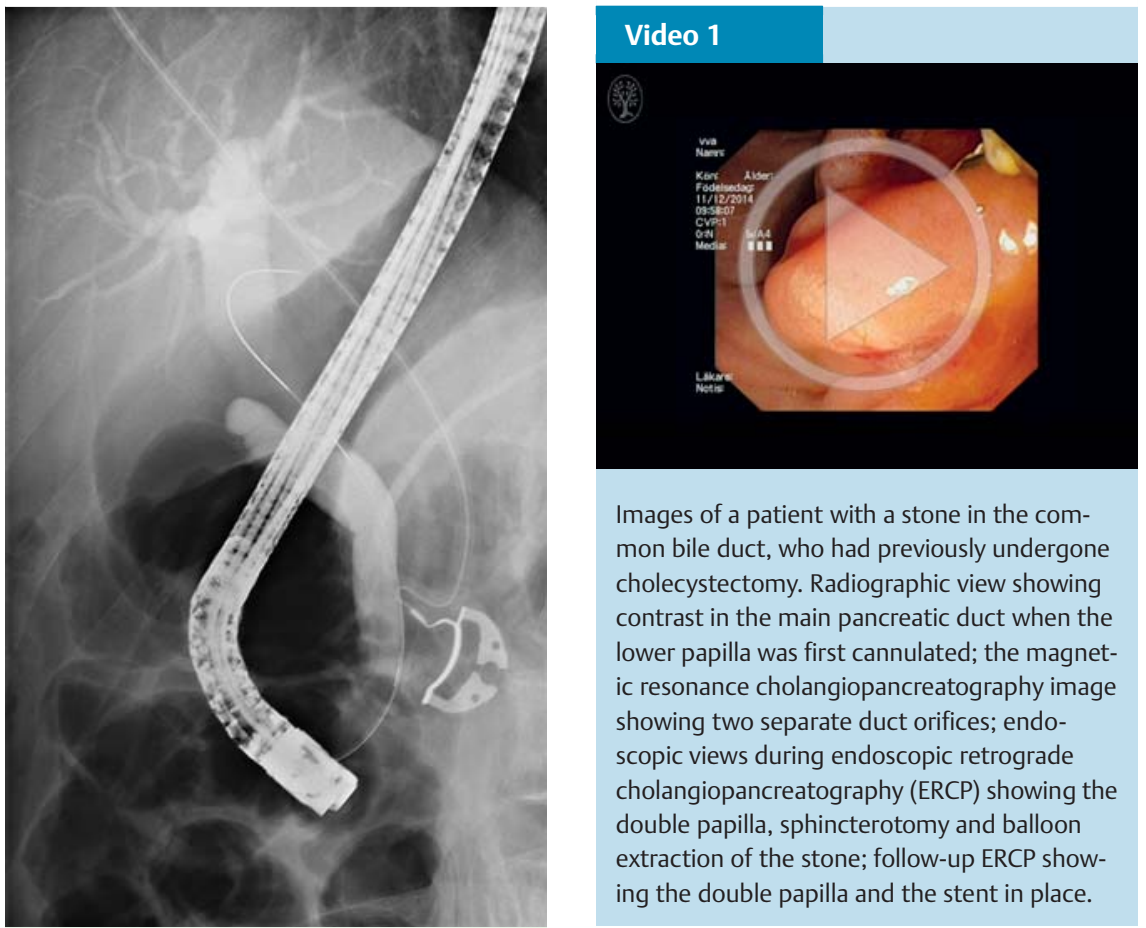

Images of a patient with a stone in the common bile duct, who had previously undergone cholecystectomy. Radiographic view showing contrast in the main pancreatic duct when the lower papilla was first cannulated; the magnetic resonance cholangiopancreatography image showing two separate duct orifices; endoscopic views during endoscopic retrograde cholangiopancreatography (ERCP) showing the double papilla, sphincterotomy and balloon extraction of the stone; follow-up ERCP showing the double papilla and the stent in place.
Dimitrios Katsarelias ${ }^{1}$, Mohammed

Faisal ${ }^{1}$, Robert Glavas ${ }^{1}$, Wolf C.

\section{Bartholomä2}

${ }^{1}$ Department of Surgery, Universitetssjukhuset i Linköping, Linköping, Sweden

${ }^{2}$ Department of Radiology, Universitetssjukhuset i Linköping, Linköping, Sweden

\section{Bibliography}

Dol http://dx.doi.org/

10.1055/s-0042-105268

Endoscopy 2016; 48: E133-E134

(c) Georg Thieme Verlag KG

Stuttgart · New York

ISSN 0013-726X

\section{Corresponding author}

\section{Dimitrios Katsarelias, MD}

Linköping University Hospital - Surgery

Linköping 58185

Sweden

Fax: +46-10-1033570

dimitrios.katsarelias@regionostergotland.se

Fig. 4 Cholangiography following contrast injection into the upper papilla. 